\title{
Transaction costs and efficiency of portfolio strategies
}

\author{
Antoon Pelsser ${ }^{\mathrm{a}, *}$, Ton Vorst ${ }^{\mathrm{b}}$ \\ "Derivative Special Trading and Research (AA 4410), ABN-AMRO Bank, P.O. Box 283, 1000 EA Amsterdam. The Netherlands \\ ' Econometric Institute, Erasmus University Rotterdam, P.O. Box 1738, 3000 DR Rotterdam, The Netherlands
}

\begin{abstract}
Some recent results for frictionless economies show that popular dynamic portfolio strategies such as stop-loss and lock-in are inefficient. I.e. for each of these strategies there exists an alternative portfolio strategy that gives the same final payoff distribution at lower initial costs. However, the alternative strategies require considerably more active trading than the simple strategies. The results rely heavily on the assumption of no transaction costs. Under this assumption the initial investment required is a linear function of the prices of the contingent claims that build the final payoff distribution. In this paper we demonstrate that, even for modest levels of transaction costs, the efficient strategies are more costly than the simple strategies, i.e. a strategy that replicates the final payoff distribution of an efficient strategy is excessively costly due to the transaction costs and the heavy trading involved. Since the initial investment is no longer a linear function of the contingent claims, the optimization problems to find the most efficient strategy are complicated combinatorial optimization problems which can only be solved for trees with a small number of steps. In a world without transaction costs, options are redundant instuments, since all payoff distributions can be replicated by trading in stocks and bonds. In the second half of this paper we show that the use of options in a world with transaction costs enables investors to realize final value distributions at lower initial costs than would be possible with trades in stocks an bonds only. Hence, although in theory options do not give rise to other portfolio strategies, they do in a more restrictive setting with transaction costs.
\end{abstract}

Keywords: Portfolio strategy; Transaction costs; Options

\section{Introduction}

In an economy without frictions, such as transaction costs, options are redundant instruments, because their payoffs can be replicated by dynamic portfolio strategies with trading only in the underlying stock and riskless bonds. Therefore, the price of an option is equal to the initial value of the portfolio strategy that replicates the option. In this setting the use of options does not create any new portfolio strategies that give rise to the same final payoffs at lower initial costs. In

\footnotetext{
* Corresponding author.
}

a more realistic case where transaction costs are incurred on trades in stocks, Leland (1985) has shown that the transaction costs in replicating an option are substantial and that large differences exist between initial values of portfolios replicating long and short positions in options. In practice options are often priced according to the Black-Scholes model which assumes no transaction costs. Hence, in a world with transaction costs, options might enhance the feasible portfolio strategies since they give payoff distributions that can only be obtained by a portfolio strategy in stocks and bonds at considerable transaction costs.

A more recent result for frictionless economies, es- 
tablished by Dybvig (1988b), is that popular dynamic portfolio strategies, such as stop-loss and lock-in, are inefficient. This means that there exists a portfolio strategy that gives the same final payoff distribution as the stop-loss strategy but has lower initial costs. The same holds for the lock-in strategy. These results depend heavily on the assumption of no transaction costs. The more efficient strategy generates more trading than the simple stop-loss strategy. Hence, in a world with transaction costs it is no longer obvious that the efficient strategies really command lower initial investments.

In this paper we investigate the following two issues:

- Are simple dynamic portfolio strategies, such as stop-loss and lock-in, really inefficient if there are transaction costs on trades of stocks?

- Does the use of options in dynamic portfolio strategies enable investors to realize final value distributions at lower costs than would be possible with only trading in stocks and bonds?

To analyze these issues we use the Cox-RossRubinstein (1979) binomial tree model for the stock prices. In the next section, we briefly describe the results of Dybvig (1988a,b) pertaining to the inefficiency of stop-loss and lock-in strategies. In Section 3 we introduce transaction costs, formulate the concepts of efficient and strongly efficient dynamic self-financing portfolio strategies and formulate optimization problems for dynamic portfolio strategies. Section 4 is devoted to the evaluation of efficient dynamic portfolio strategies when there are transaction costs, while Section 5 addresses the question of whether options enhance the possibilities of investors to achieve certain payoff distributions at low costs. The last section concludes the paper and offers some directions for further research.

\section{The Dybvig model}

In this section, we briefly outline the payoff distribution pricing model (PDPM) for the binomial asset pricing model as described in Dybvig (1988b). Fig. 1 represents a specific (4-period) example of the wellknown binomial model of stock returns introduced by Cox, Ross and Rubinstein (1979). The initial stock price $S_{0}$ is equal to 16 , one plus the riskless interest rate is equal to one (hence, the riskless interest rate is zero) and the stock price doubles or halves each period, with probability $1 / 2$. Since we are interested in both path-dependent trading strategies and transaction costs, we do not give the standard (recombined) binomial tree, but the expanded tree. In fact, the expanded tree properly shows the increasing sequence of $\sigma$-algebras that describes the information structure of the model.

The final states are numbered $\omega_{1}, \ldots, \omega_{16}$. Since the binomial model is complete, each contingent claim that gives payoffs in the final period can be duplicated by a self-financing trading strategy. If there are no arbitrage possibilities, the initial outlay for this strategy must be the price of the contingent claim. The price of a contingent claim that pays 1 in a given state and 0 in all other states is called the state price of the given state. For a given contingent claim its price is the sum over all states of the payment in that state times the state price. In fact, the state price is equal to the probability of the state under the unique martingale measure of the pricing model.

Next, consider the stop-loss strategy where we initially buy one stock and sell the stock for bonds as soon as the stock price drops to 8 . From here onward we deal solely with bonds. In Fig. 2 this strategy is illustrated such that at each node in the tree the first number specifies the number of stocks in the portfolio and the second specifies the amount invested in bonds. Furthermore, the final value of the portfolio at maturity in each state is given. In Fig. 3 an alternative to the stop-loss strategy is given. At each node, we again specify the number of stocks in the portfolio, and the amount invested in bonds. The alternative strategy is self-financing and has the same payoff distribution as the stop-loss strategy. Both strategies give a payoff of 8 with probability $10 / 16$, a payoff of 16 with probability $2 / 16$, a payoff of 64 with probability $3 / 16$ and a payoff of 256 with probability $1 / 16$. However, the initial outlay of the alternative strategy is only $15 \frac{65}{81}$, i.e. $0.938 \times 16=15.01$ for the stock plus 0.79 for the bond. The reduction in price of the alternative strategy is due to the fact that the payoffs in states $\omega_{6}$ and $\omega_{9}$ are interchanged. The alternative strategy gives a higher payoff in a state with a lower state price and a lower payoff in a state with a higher price. The reduction in price is equal to the product of the difference in payoffs multiplied by the difference in state prices: 


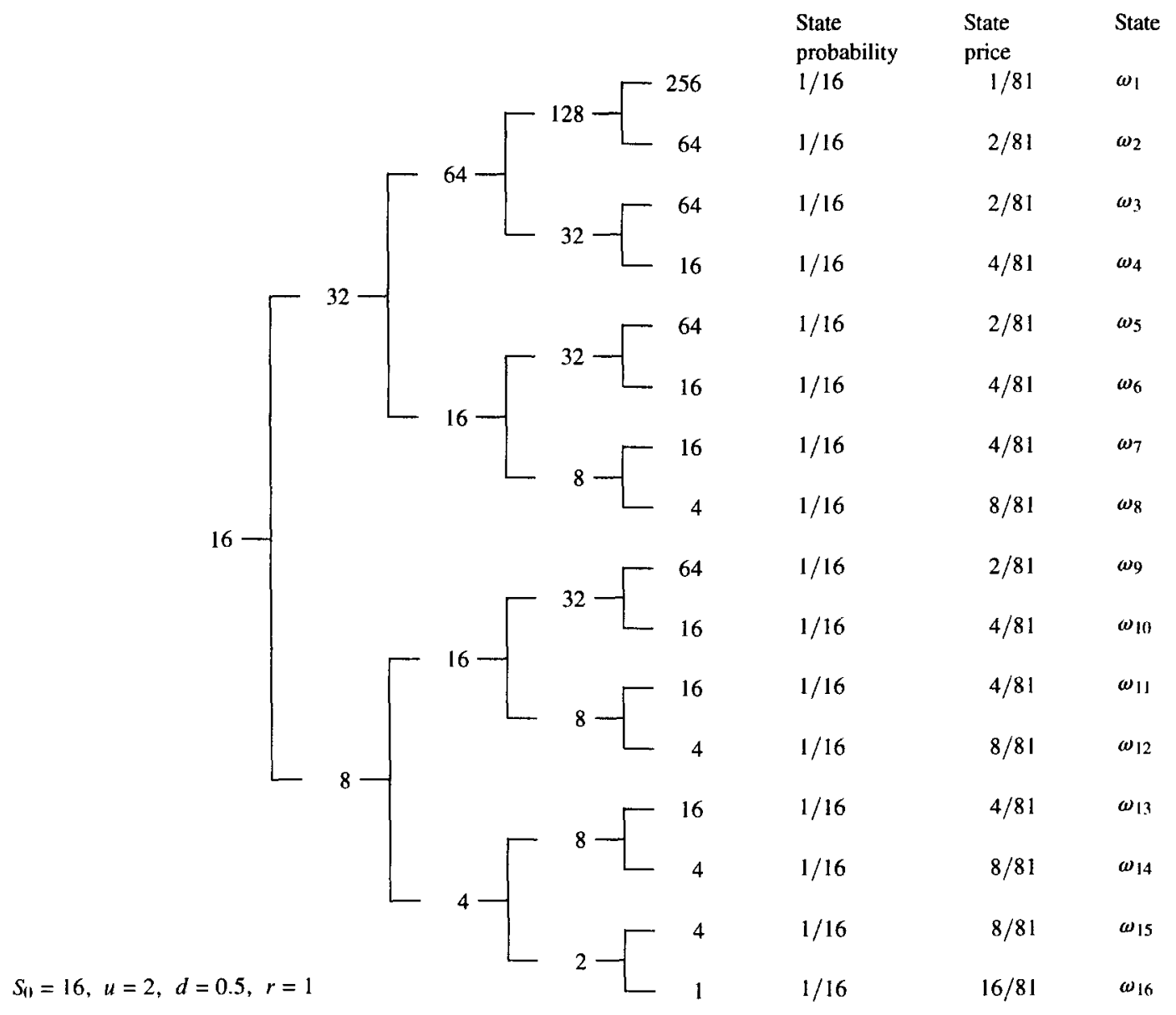

Fig. 1. Expanded binomial tree.

$(16-8) \times(4 / 81-2 / 81)=16 / 81$

It is clear from the above example that an individual who maximizes expected utility of terminal wealth with a strictly increasing utility function prefers the alternative strategy to the stop-loss strategy. Dybvig (1988a) proves that the following properties of portfolio strategies are equivalent:

- The portfolio strategy $c$ is chosen by some agent who has strictly increasing von NeumanMorgenstern preferences over terminal wealth;

- The final portfolio value is non-increasing in the terminal state-price. ${ }^{1}$

\footnotetext{
' In fact, Dybvig's result is more general. The second property should be that the final portfolio value is non-increasing in the terminal state price density. The terminal state-price density can be obtained by dividing the state price by the state probability. The state price density is the likelihood ratio process or the risk
}

Portfolio strategies that have the second property are called efficient, while other portfolio strategies will be called inefficient. If we compare the final portfolio values of the alternative strategy with the state price, it is clear that this strategy is efficient.

In Dybvig (1988b), the simple example above is extended to a binomial model with more trading intervals and non-zero interest rates. Dybvig calculates that the inefficiency of the stop-loss strategy might amount to 100 basispoints for an investment period of 1 year with a risk-free interest rate of $8 \%$, a stock with an expected return of $16 \%$ and a volatility of $20 \%$ and

adjustment process (see e.g. Dothan (1990) for an elaboration on the state price density). Since, in our case all terminal states have the same probability, state prices and state price densities only differ by a constant factor. 


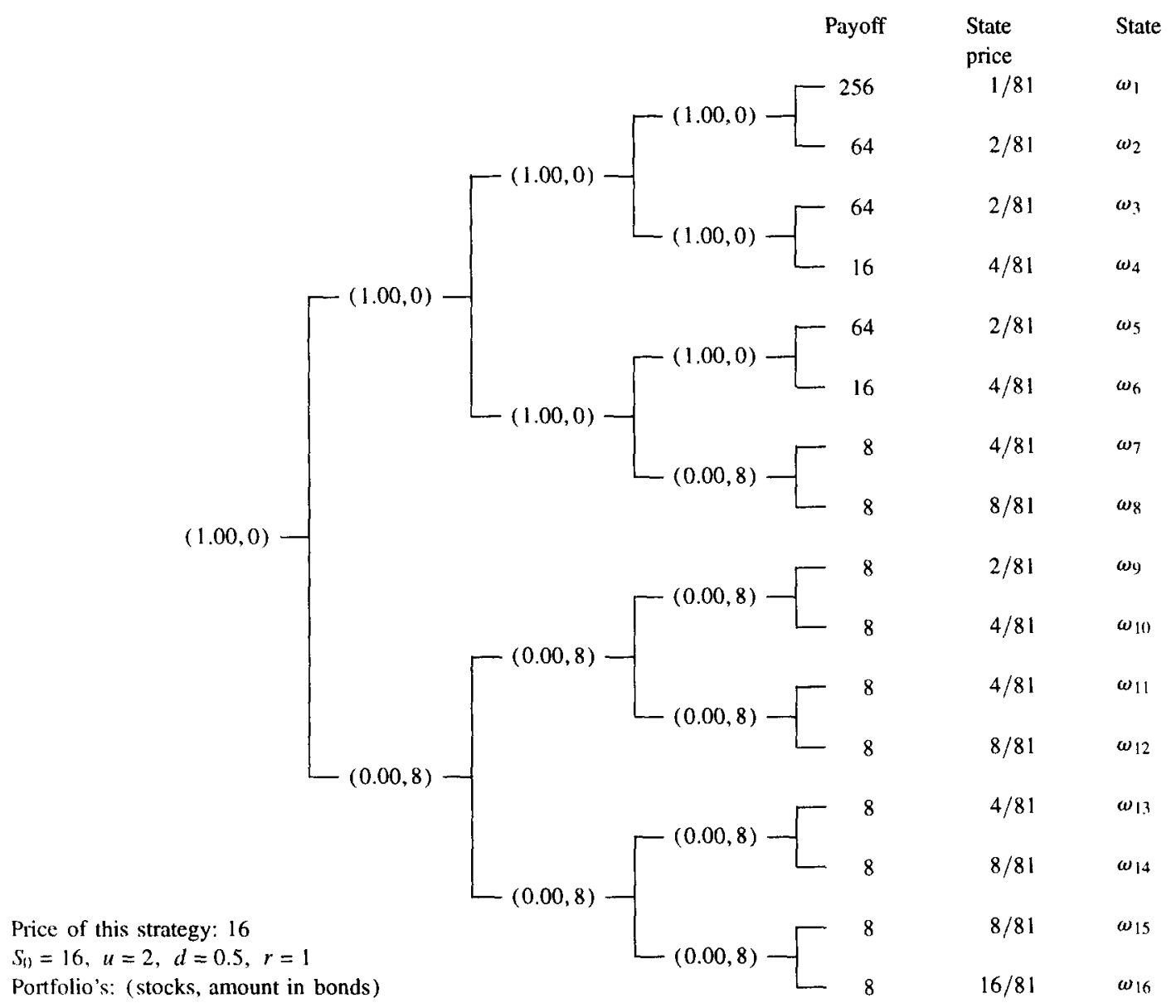

Fig. 2. Stop-loss strategy.

a binomial tree of 360 trading intervals. This means there exists an alternative portfolio strategy with the same final payoff distribution as the stop-loss strategy that requires an initial outlay that is $1 \%$ lower.

In the binomial model, with upward and downward probability both equal to $1 / 2$, the state price is a function of the final stock price. If two final states have the same stock price they will also have the same state price. If the expected return on the stock is higher then the risk-free return, then this function is strictly decreasing. Hence, a portfolio strategy is efficient if and only if the final portfolio value is a non-decreasing function of the final stock price. This implies that a portfolio that consists of only holding a put option is inefficient. However, a stock plus a put-option on that stock is an efficient strategy. A call option itself is also an efficient strategy and it is clear that lock-in strate- gies, where the stock is sold if its price rises to a given level, are inefficient.

Nowadays, fund managers not only consider the risk and return of a portfolio strategy, but also compare the performance of their portfolio with a benchmark index (e.g. Roll, 1992). If we replace the stock with the index in the model above, this implies that efficient strategies will have a final portfolio value that is increasing in the value of the index. This can be interpreted as efficient strategies being those strategies with the lowest trading error given a final portfolio value distribution function. 


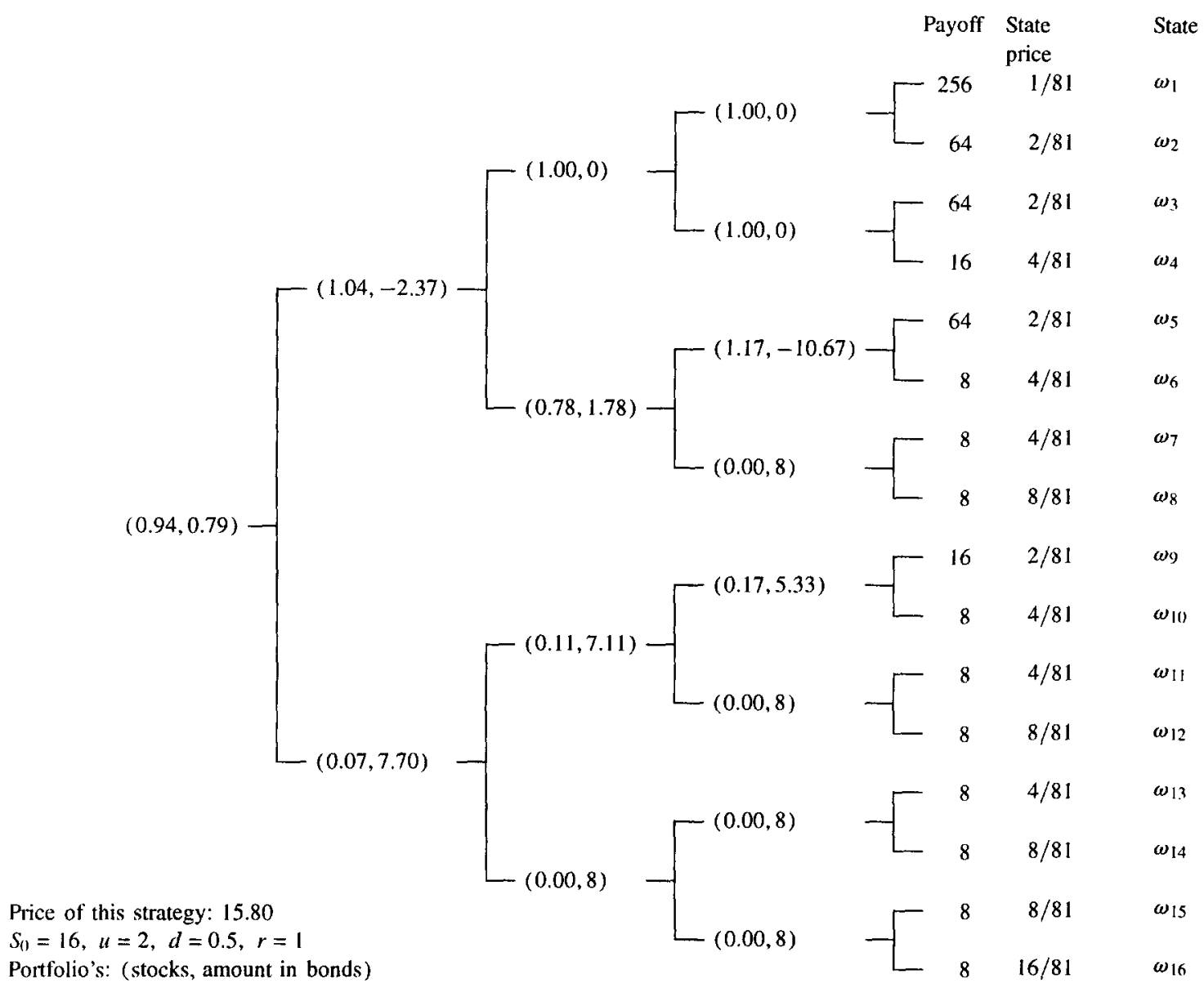

Fig. 3. Alternative stop-loss strategy.

\section{The influence of transaction costs}

If we compare Figs. 2 and 3, we see that although both strategies differ only in portfolio values in two states, the holdings in bonds and stocks differ substantially between the two strategies. For the stop-loss strategy we only have to sell the stock and buy the bond in some cases. For the alternative strategy the portfolio must be adjusted in each period, whatever happens with the stock value. Hence, it is not unlikely that the alternative strategy generates higher transaction costs. In this section we model these transaction costs. To a large extent we follow the notation from Bensaid, Lesne, Pagès and Scheinkman (1992).

There are $T+1$ dates, $t=0,1, \ldots, T$. The state space $\Omega=\{u, d\}^{T}$ is the set of paths in an expanded binomial tree, where $u$ stands for an up-state move- ment and $d$ for a down-state movement. It is assumed that at each step the probability of an up-state is $1 / 2$. A path is denoted by $\omega=\left(\omega_{1}, \ldots, \omega_{t}, \ldots, \omega_{T}\right)$ with $\omega_{i} \in\{u, d\} . \omega^{t}=\left(\omega_{1}, \ldots, \omega_{t}\right)$ will denote a path up to time $t$ and $\mathcal{F}_{t}$ is the $\sigma$-algebra of information up to time $t$. There is a risky asset called stock and a riskless asset called bond. The one-period return on the riskless bond is constant over time and states. One plus the riskless return will be denoted by $r$. The value of the stock at time $t$ and state $\omega$ is denoted by $S_{t}(\omega)$, which is an adapted process. A dynamic portfolio strategy is defined by two $\mathcal{F}$-adapted processes $\Delta$ and $B$. $\Delta_{t}$ is the number of stocks that is held between date $t$ and $t+1$ and $B_{t}$ is the amount of dollars invested in the riskless bond during the same period. Transaction costs are due on trades in stocks and as in Boyle and Vorst (1992) they are proportional to the amount 
traded. Let $k$ be the proportion of transaction costs. A dynamic portfolio strategy is called self-financing with transaction costs $k$ (or shortly self-financing) if the following equation holds

$$
\begin{gathered}
\left(\Delta_{t-1}(\omega)-\Delta_{t}(\omega)\right) S_{t}(\omega)+r B_{t-1}(\omega)-B_{t}(\omega) \\
=k\left|\Delta_{t-1}(\omega)-\Delta_{t}(\omega)\right| S_{t}(\omega) \\
\forall t \leqslant T-1, \forall \omega \in \Omega .
\end{gathered}
$$

The final payoff $V$ of a portfolio strategy is given by $V(\omega)=\Delta_{T-1} S_{T}(\omega)+r B_{T-1}(\omega)$, which is a realvalued random variable on $\Omega$. Let

$$
\begin{gathered}
G(\Delta, B)(x)=\operatorname{Pr}\left\{\omega \in \Omega \mid \Delta_{T-1} S_{T}(\omega)+r B_{T-1}(\omega)\right. \\
\leqslant x\}
\end{gathered}
$$

be the probability distribution function of this random variable. We call $G(\Delta, B)$ the payoff distribution function. Note that $\Delta_{0}$ and $B_{0}$ do not depend on $\omega$ since $(\Delta, B)$ is adapted. $\Delta_{0} S_{0}+B_{0}$ is called the initial cost of the strategy.

Definition 1. Let the transaction costs be $k$. A dynamic self-financing portfolio strategy $(\Delta, B)$ is efficient if there is no other dynamic self-financing portfolio strategy $\left(\Delta^{\prime}, B^{\prime}\right)$ such that:
(i) $G\left(\Delta^{\prime}, B^{\prime}\right)=G(\Delta, B)$;
(ii) $\Delta_{0}^{\prime} S_{0}+B_{0}^{\prime}<\Delta_{0} S_{0}+B_{0}$.

In this terminology the stop-loss strategy of Section 2 is inefficient if there are no transaction costs, since the alternative strategy has the same payoff distribution function and has lower initial costs.

With transaction costs one can define a stronger form of efficiency similar to the concepts in Edirisinghe, Naik and Uppal (1993) and Bensaid, Lesne, Pagès and Scheinkman (1992) for replicating portfolio strategies. According to Ingersoll (1987) a payoff distribution function $G\left(\Delta^{\prime}, B^{\prime}\right)$ stochastically dominates another payoff distribution function $G(\Delta, B)$ if $G\left(\Delta^{\prime}, B^{\prime}\right)(x) \leqslant G(\Delta, B)(x)$ for all $x \in \mathbb{R}$ with strict inequality for at least one $x \in \mathbb{R}$.

Definition 2. Let the transaction costs be $k$. A dynamic self-financing portfolio strategy $(\Delta, B)$ is strongly efficient if it is efficient and there exists no other dynamic self-financing portfolio strategy $\left(\Delta^{\prime}, B^{\prime}\right)$ such that:

(i) $G\left(A^{\prime}, B^{\prime}\right)$ stochastically dominates $G(A, B)$;

(ii) $\Delta_{0}^{\prime} S_{0}+B_{0}^{\prime} \leqslant \Delta_{0} S_{0}+B_{0}$.

Bensaid, Lesne, Pagès and Scheinkman (1992) relax the self-financing condition (3.1) to

$$
\begin{gathered}
\left(\Delta_{t-1}(\omega)-\Delta_{t}(\omega)\right) S_{t}(\omega)+r B_{t-1}(\omega)-B_{t}(\omega) \\
\geqslant k\left|\Delta_{t-1}(\omega)-\Delta_{t}(\omega)\right| S_{t}(\omega) \\
\forall t \leqslant T-1, \forall \omega \in \Omega .
\end{gathered}
$$

This means that after each trade some non-negative payoff remains which can be used for consumption if positive. However, when these positive payoffs are invested in risk-less bonds until maturity, the final payoff distribution stochastically dominates the final payoff distribution where the positive payoffs are immediately consumed. Hence, if one is checking for strong efficiency and one finds a dynamic portfolio strategy $\left(\Delta^{\prime}, B^{\prime}\right)$ that satisfies (3.3) and conditions (i) and (ii) of Definition 2, then there also exists a dynamic self-financing portfolio strategy that satisfies (i) and (ii).

If there are no transaction costs then efficiency implies strong efficiency. Let $(A, B)$ be an efficient strategy and assume there exists a strategy $\left(\Delta^{\prime}, B^{\prime}\right)$ that satisfies (i) and (ii) of Definition 2. Both distribution functions $G(\Delta, B)$ and $G\left(\Delta^{\prime}, B^{\prime}\right)$ are stepfunctions, since there are only finitely many possible portfolio values. In Appendix A, it is shown that there exists a dynamic self-financing strategy $\left(\Delta^{\prime \prime}, B^{\prime \prime}\right)$ that has non-negative payoffs in all states and a positive payoff in at least one state such that $G(\Delta, B)=G\left(\Delta^{\prime}-\Delta^{\prime \prime}, B^{\prime}-B^{\prime \prime}\right)$. Since there are no transaction costs $\left(\Delta^{\prime}-\Delta^{\prime \prime}, B^{\prime}-B^{\prime \prime}\right)$ is self-financing. By the no-arbitrage assumption $\Delta_{0}^{\prime \prime} S_{0}+B_{0}^{\prime \prime}>0$ and hence $\left(\Delta^{\prime}-\Delta^{\prime \prime}, B^{\prime}-B^{\prime \prime}\right)$ is more efficient than $(\Delta, B)$ in the sense of Definition 1, which gives the required contradiction. Essential in this argument is that the difference between two self financing strategies is again self-financing. This doesn't hold if there are transaction costs.

To check whether a dynamic self-financing portfolio strategy with non-zero transaction costs is efficient there is no easy criterion such as the one described in the previous paragraph for the no transaction costs case. Let $(\Delta, B)$ be a dynamic self-financing 


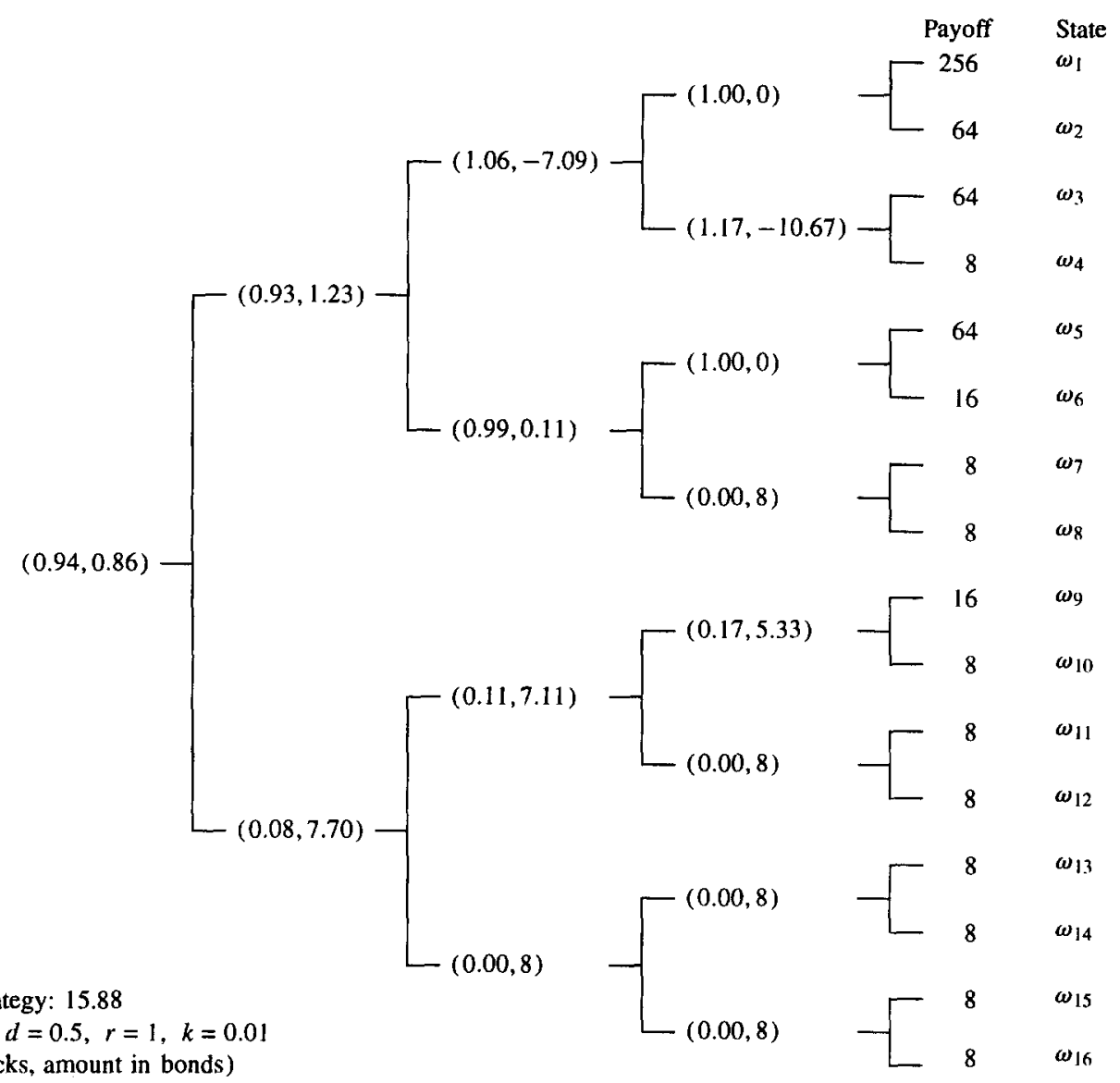

Price of this strategy: 15.88

$S_{0}=16, u=2, d=0.5, r=1, k=0.01$

Portfolio's: (stocks, amount in bonds)

Fig. 4. Strongly efficient stop-loss strategy with transaction costs.

portfolio-strategy. One might try to find the dynamic self-financing portfolio strategy $\left(\Delta^{\prime}, B^{\prime}\right)$ among those strategies for which the final payoff distribution dominates $G(\Delta, B)$ and that has the lowest initial costs. This problem can be formulated as a mixed-integer programming problem, as is shown in Appendix B, and its solution will be a strongly efficient dynamic portfolio strategy. However, the complexity of this problem allows one only to find solutions for small values of $T$. In Fig. 4 the optimal strategy in the 4 period case is given where transaction costs are $1 \%$ and the strategy must dominate the final payoff distribution of a stop-loss strategy with no transaction costs. Hence, the final payoff should be at least 256 with a probability of $1 / 16,64$ with a probability of $4 / 16,16$ with a probability of $6 / 16$, and always exceed 8 .

Fig. 5 gives the replicating portfolio for the alterna- tive strategy of Fig. 3 with $1 \%$ transaction costs. Figs. 4 and 5 have interchanged payoffs in states $\omega_{4}$ and $\omega_{6}$. The strategy given in Fig. 5 has the cheapest initial costs of all strategies that generate the payoff random variable of Fig. 5. This will be seen more clearly in the next section, where it is shown that there exists a unique "cheapest" strategy that generates this payoff random variable. However, we see that the initial cost of this strategy is higher. In the no transaction costs case both payoff schemes would have the same price, since we interchanged only the payoffs between states with the same state-price density. Hence, with transaction costs we might not only have to change payoffs between states with different state-price densities, but also between states with the same state-price densities. This illustrates the complexity of the optimization problem with transaction costs. 


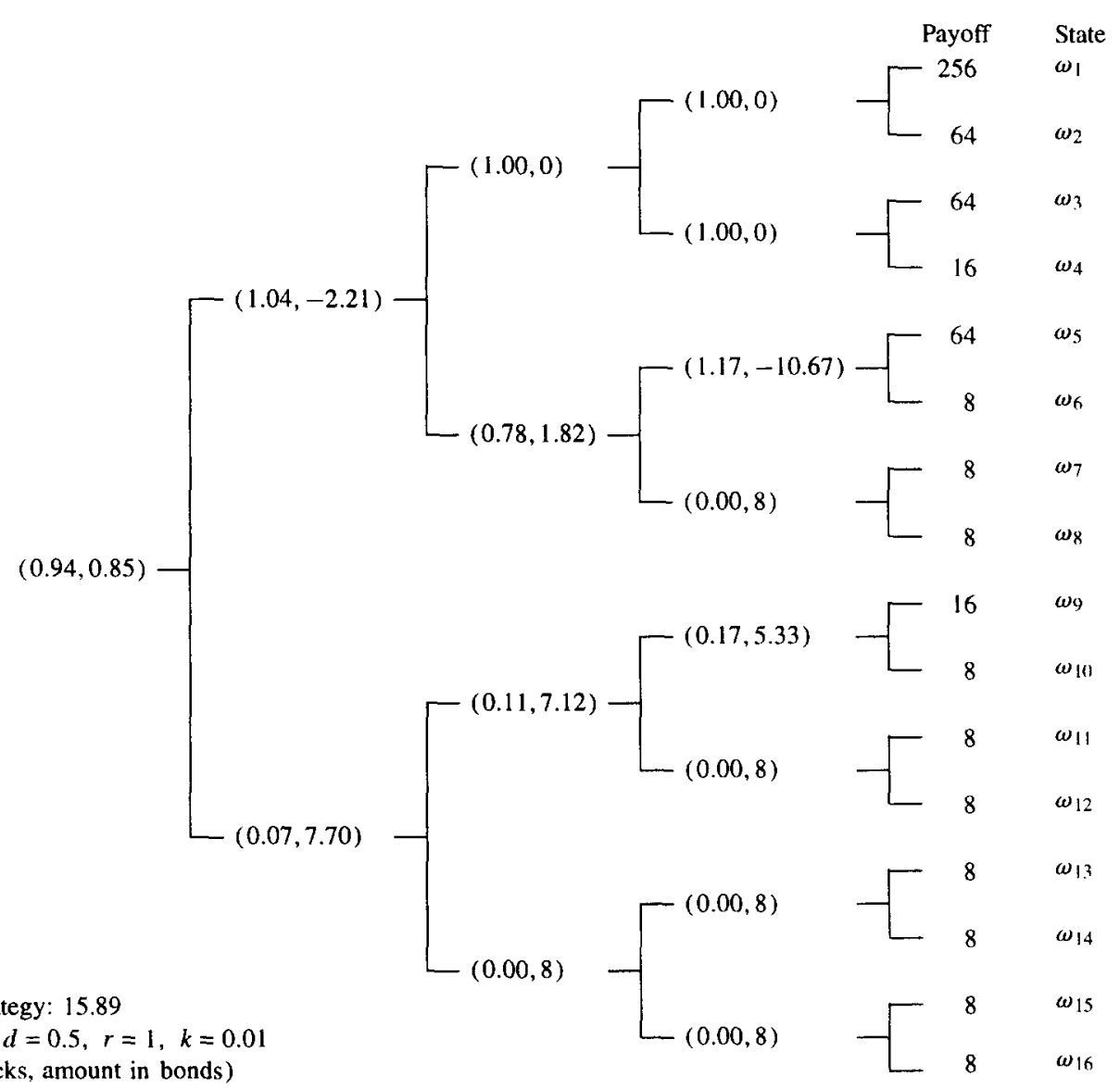

Price of this strategy:
$S_{(1}=16, u=2, d=0.5, r=1, k=0.01$

Portfolio's: (stocks, amount in bonds)

Fig. 5. Alternative stop-loss strategy with transaction costs.

\section{Dybvig's efficient strategies and transaction costs}

In this section, we compare the efficient strategies as described in Section 2 with the inefficient stoploss strategies in the presence of transaction costs. Let $(\Delta, B)$ represent a dynamic portfolio strategy that is self-financing if there are no transaction costs. To evaluate how this strategy is affected by transaction costs, we use the following result, based on the proof of Theorem 4 of Boyle and Vorst (1992), for small values of $k$.

Theorem 3. If the transaction costs $k$ on stocks satisfy the following condition

$(1+k) d<(1-k) u$, then there exists a unique dynamic portfolio strategy $\left(\Delta^{k}, B^{k}\right)$ that is self-financing for transaction costs $k$ such that

$$
\begin{aligned}
& \Delta_{T}(\omega) S_{T}(\omega)+B_{T}(\omega)=\Delta_{T}^{k}(\omega) S_{T}(\omega)+B_{T}^{k}(\omega) \\
& \forall \omega \in \Omega .
\end{aligned}
$$

Furthermore, $\left(\Delta^{k}, B^{k}\right)$ can be explicitly found by solving equations (3.1) backwards through the expanded binomial tree.

Proof. In each node of the tree at time $t$, there are two equations (3.1), one for the upward-move and one for the downward move. The unknowns are $\Delta_{t}(\omega)$ and $B_{t}(\omega)$. It follows from the proof of Theorem 4 of Boyle and Vorst (1992) that these two equations with two unknowns have a unique solution if condition (4.1) is satisfied. These unique solu- 


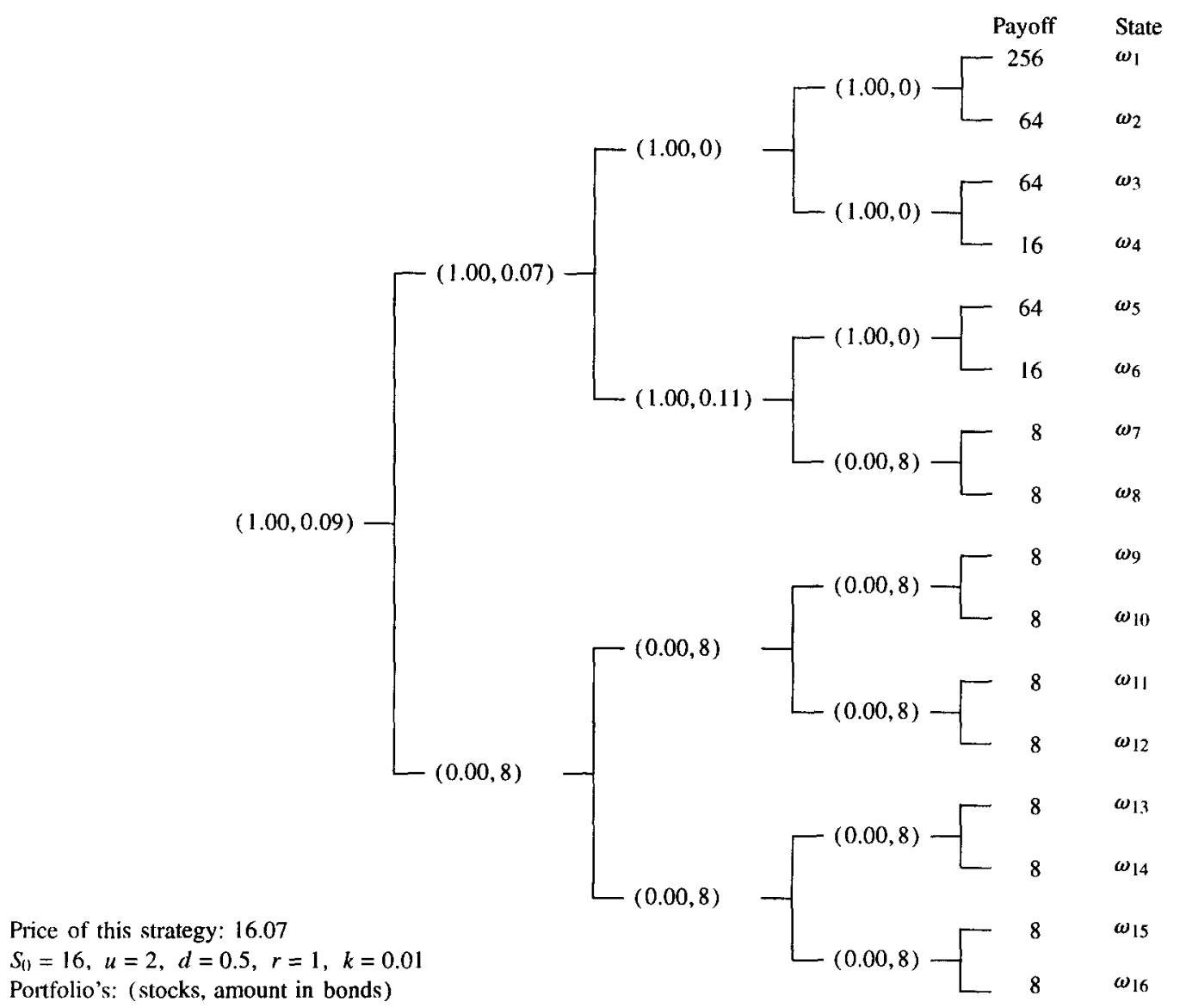

Fig. 6. Stop-loss strategy with transaction costs.

tions can be calculated backwards to find the strategy $\left(\Delta^{k}, B^{k}\right)$.

This theorem allows us to calculate the price of the initial portfolio. This is necessary to be able to perform the strategy under transaction costs in order to obtain the final portfolio $\left(\Delta_{T}, B_{T}\right)$. Fig. 6 gives the strategy $\left(\Delta^{0.01}, B^{0.01}\right)$ for the stop-loss strategy, while in Fig. 5 the strategy $\left(\Delta^{0.01}, B^{0.01}\right)$ is given for the efficient strategy from Fig. 3. (Hence the transaction costs are $1 \%$.) We see that the efficient strategy from Fig. 3 has higher total transaction costs $(15.89-15.80=0.09)$ than the stop-loss strategy $(16.06-16.00=0.06)$.

However, the efficient strategy still has lower initial costs and is thus more efficient than the stop-loss strategy in this simple example. To investigate the influence of transaction costs further, we have to ex- pand the model to more trading intervals, where the changes in stock prices are smaller. Since in each step the number of nodes is doubled in an expanded tree, one can only use a modest number of trading intervals. In Table 1 we give the initial costs and portfolio compositions both for the stop-loss strategy and the alternative efficient strategy for 18 trading intervals and for several levels of transaction costs. In this example, the values of the volatility, interest rates, time to maturity and expected return on the stock are equal to the values used in Dybvig (1988b). The stop-loss value is 14.4 , which is $90 \%$ of the original portfolio value. Hence, if the stock price falls below the discounted value of 14.4 , the stock is sold for bonds. We see from Table 1 that not only do the total transaction costs differ but also that the so-called efficient dynamic portfolio strategy now requires higher initial 
Table I

Prices of replicating strategies using stocks

\begin{tabular}{|c|c|c|c|c|c|c|c|c|}
\hline \multirow{3}{*}{$\begin{array}{l}k \\
0 \%\end{array}$} & \multicolumn{4}{|c|}{ Stop-loss at 14.4} & \multicolumn{4}{|c|}{ Lock-in at 18.4} \\
\hline & \multicolumn{2}{|c|}{ Normal } & \multicolumn{2}{|c|}{ Efficient } & \multicolumn{2}{|c|}{ Normal } & \multicolumn{2}{|c|}{ Efficient } \\
\hline & 16.00 & $\begin{array}{l}1.0000 \mathrm{~s} \\
0.0000 \mathrm{~b}\end{array}$ & 15.97 & $\begin{array}{l}0.8849 \mathrm{~s} \\
1.8119 \mathrm{~b}\end{array}$ & 16.00 & $\begin{array}{l}1.0000 \mathrm{~s} \\
0.0000 \mathrm{~b}\end{array}$ & 15.89 & $\begin{array}{l}0.5796 \mathrm{~s} \\
6.6185 \mathrm{~b}\end{array}$ \\
\hline $0.5 \%$ & 16.02 & $\begin{array}{l}0.9906 \mathrm{~s} \\
0.1732 \mathrm{~b}\end{array}$ & 16.10 & $\begin{array}{l}0.8726 \mathrm{~s} \\
2.1339 \mathrm{~b}\end{array}$ & 16.06 & $\begin{array}{r}1.0188 \mathrm{~s} \\
-0.2420 \mathrm{~b}\end{array}$ & 16.10 & $\begin{array}{l}0.5534 \mathrm{~s} \\
7.2484 \mathrm{~b}\end{array}$ \\
\hline $1 \%$ & 16.06 & $\begin{array}{l}0.9817 \mathrm{~s} \\
0.3420 \mathrm{~b}\end{array}$ & 16.19 & $\begin{array}{l}0.8622 \mathrm{~s} \\
2.3965 \mathrm{~b}\end{array}$ & 16.12 & $\begin{array}{r}1.0362 \mathrm{~s} \\
-0.4541 \mathrm{~b}\end{array}$ & 16.34 & $\begin{array}{l}0.5152 \mathrm{~s} \\
8.0920 \mathrm{~b}\end{array}$ \\
\hline $2 \%$ & 16.11 & $\begin{array}{l}0.9656 \mathrm{~s} \\
0.6591 \mathrm{~b}\end{array}$ & 16.53 & $\begin{array}{l}0.8413 \mathrm{~s} \\
2.8902 \mathrm{~b}\end{array}$ & 16.28 & $\begin{array}{r}1.0737 \mathrm{~s} \\
-0.8994 \mathrm{~b}\end{array}$ & 16.92 & $\begin{array}{r}0.3355 \mathrm{~s} \\
11.5482 \mathrm{~b}\end{array}$ \\
\hline
\end{tabular}

$S_{10}=16, \sigma=20 \%, \mu=16 \%, R=8 \%, T=1,18$ trading intervals.

total costs than the simple stop-loss strategy, even if the transaction costs on stocks are as low as $0.5 \%$. We conjecture that the effect is more pronounced if we extend to even more trading intervals. Hence, we can conclude that the so-called efficient dynamic portfolio strategy is less efficient than the stop-loss strategy in the presence of transaction costs.

The initial costs of a lock-in strategy are also given in Table 1. If an investor follows a lock-in strategy, he intially buys a stock and holds the stock until it reaches a certain pre-specified level (in our case the discounted value of 18.4). At that moment the stock is sold and the revenues are invested in riskless bonds. Hence, a lock-in strategy is similar to a stop-loss strategy, differing only in the pre-specified level. For the lock-in strategy the pre-specified level lies above the intial stock price, while for the stop-loss strategy, it lies below the initial stock-price. Without transaction costs the lock-in strategy is also inefficient and we see from Table 1 that there exists an efficient strategy that requires only an initial investment of 15.89 . This strategy was also constructed using Dybvig's method described in Section 2. In the presence of transaction costs we see that the efficient strategy becomes costly. Therefore it requires substantially higher initial investments than the lock-in strategy and hence is no longer efficient. Thus, the general conclusion is the same for this strategy as for the stop-loss strategy.

\section{Options and cost reduction in portfolio strategies}

In the absence of transaction costs the possibility to trade in options does not add extra opportunities in the binomial tree model. If options are priced arbitragefree then all portfolio strategies that can be executed with bonds, shares of the stock and options on the stock, can also be executed with only bonds and shares, with the same costs. Hence, options are redundant assets. In this section we investigate whether options add extra possibilities or reduce costs in the case where transaction costs are present.

We assume that options are priced at their CoxRoss-Rubinstein (CRR) binomial tree value, even in the case that there are transaction costs for trading in stocks. Therefore, we do not use the pricing methodology of Boyle and Vorst (1992), which prices long and short option positions using a replicating strategy. We assume that there is an equilibrium between demand and supply in the option markets such that the price of options is the no transaction costs replicating price. $^{2}$ This implies that we do not introduce arbitrage opportunities, since the costs of replicating a long option with transaction costs are higher than the market price of this option. For a short option, the

\footnotetext{
${ }^{2}$ Alternatively stated: all traders in the market use a CRR model to establish a fair price for options.
} 
Table 2

Prices of replicating strategies using options

\begin{tabular}{|c|c|c|c|c|c|c|c|c|}
\hline \multirow{3}{*}{$\frac{l}{0 \%}$} & \multicolumn{4}{|c|}{ Stop-loss at 14.4} & \multicolumn{4}{|c|}{ Lock-in at 18.4} \\
\hline & \multicolumn{2}{|l|}{ Normal } & \multicolumn{2}{|c|}{ Efficient } & \multicolumn{2}{|l|}{ Normal } & \multicolumn{2}{|l|}{ Efficient } \\
\hline & 16.00 & $\begin{array}{r}1.0064 \mathrm{c} \\
10.1110 \mathrm{~b}\end{array}$ & 15.97 & $\begin{array}{r}0.8906 \mathrm{c} \\
10.7594 \mathrm{~b}\end{array}$ & 16.00 & $\begin{array}{r}-1.1035 p \\
21.9204 b\end{array}$ & 15.89 & $\begin{array}{r}-0.6369 p \\
19.3241 \mathrm{~b}\end{array}$ \\
\hline $0.5 \%$ & 16.01 & $\begin{array}{r}1.0039 \mathrm{c} \\
10.1309 \mathrm{~b}\end{array}$ & 16.02 & $\begin{array}{r}0.8890 \mathrm{c} \\
10.8139 \mathrm{~b}\end{array}$ & 16.02 & $\begin{array}{r}-1.1048 \mathrm{p} \\
21.9501 \mathrm{~b}\end{array}$ & 15.98 & $\begin{array}{r}-0.6135 p \\
19.2677 b\end{array}$ \\
\hline $1 \%$ & 16.01 & $\begin{array}{r}1.0014 c \\
10.1504 b\end{array}$ & 16.06 & $\begin{array}{r}0.8874 \mathrm{c} \\
10.8629 \mathrm{~b}\end{array}$ & 16.05 & $\begin{array}{r}-1.1061 \mathrm{p} \\
21.9796 \mathrm{~b}\end{array}$ & 16.06 & $\begin{array}{r}-0.5847 \mathrm{p} \\
19.1972 \mathrm{~b}\end{array}$ \\
\hline $2 \%$ & 16.02 & $\begin{array}{r}0.9969 \mathrm{c} \\
10.1882 \mathrm{~b}\end{array}$ & 16.12 & $\begin{array}{r}0.8843 \mathrm{c} \\
10.9478 \mathrm{~b}\end{array}$ & 16.09 & $\begin{array}{r}-1.1086 \mathrm{p} \\
22.0386 \mathrm{~b}\end{array}$ & 16.25 & $\begin{array}{r}-0.5134 p \\
18.9999 b\end{array}$ \\
\hline
\end{tabular}

c: call option with exercise price 11 for stop-loss strategy.

p: put option with exercise price 23 for lock-in strategy.

$S_{()}=16, \sigma=20 \%, \mu=16 \%, R=8 \%, T=1,18$ trading intervals.

costs are lower. However, we assume that there are proportional transaction costs associated with trades in options. Let $l$ be the proportionality factor, which can be different from $k$, the proportional transaction costs for trades in stocks. Let $C_{t}(\omega)$ be the price of a specific option on the stock with expiration date $T$. A dynamic self-financing trading strategy in this option consists of two $\mathcal{F}$-adapted processes $\Gamma$ and $B$ such that the following equation holds

$$
\begin{aligned}
& \left(\Gamma_{t-1}(\omega)-\Gamma_{t}(\omega)\right) C_{t}(\omega)+r B_{t-1}(\omega)-B_{t}(\omega) \\
& =l\left|\Gamma_{t-1}(\omega)-\Gamma_{t}(\omega)\right| C_{t}(\omega) \\
& \quad \forall t \leqslant T-1, \forall \omega \in \Omega .
\end{aligned}
$$

$\Gamma_{t}(\omega)$ is the number of options in the portfolio during the period from $t$ to $t+1$, while $B_{t}(\omega)$ is the amount invested in riskless bonds during the same period. One can define efficient and strongly efficient strategies for options similar to the concepts for stocks as defined in Section 2. However, to find optimal strategies one would run into the same complexity problems as in the case of stocks. Therefore, our goal in this section is more modest. We want to investigate whether options enable us to replicate the same payoff schemes with lower initial costs. First, we need to investigate whether trading in the option allows us to attain every arbitrary payoff function $V(\omega)$ as is the case for stocks if condition (4.1) is met. Similar to the proof of the theorem of Section 4, one can show that if

$$
(1+l) C_{t+1}(\tilde{\omega})<(1-l) C_{t+1}(\omega)
$$

for all $t \leqslant T-1$ and all states $\omega, \tilde{\omega}$ that are identical except for the period $t+1$, where $\tilde{\omega}$ is the state in which the stock price path moves downward during this period and $\omega$ the the state in which the path moves upward ${ }^{3}$, then all payoff functions can be replicated.

For most options, condition (5.2) will not be satisfied for all $t \leqslant T-1$ and all states. For example, if the option is far out-of-the-money at some date and at some specific state (for a call option in one of its lower states) it will have a zero value not only at that state, but also at the next period in both possible states. In that case, condition (5.2) is not fulfilled. Hence, with most options we cannot replicate all payoff schemes. But condition (5.2) is only a sufficient condition to replicate all payoff schemes. It is therefore certainly possible that a specific payoff scheme can be replicated by a specific option. For example, in the payoff scheme of the stop-loss strategy in Fig. 2 all payoffs in the lower part of the expanded tree are equal to 8 . A call option with exercise price 16 will have value zero

\footnotetext{
${ }^{3}$ Using the notation introduced in Section 3 we can write $\omega=\left(\omega_{1}, \ldots, \omega_{t}, u, \omega_{t+2}, \ldots, \omega_{T}\right)$ and $\tilde{\omega}=\left(\omega_{1}, \ldots, \omega_{t}, d\right.$, $\left.\omega_{t+2}, \ldots, \omega_{T}\right)$.
} 
at states $\omega_{13}, \omega_{14}, \omega_{15}, \omega_{16}$ at $t=2,3$ and 4 . However, with this option one can still replicate the stop-loss scheme since in these states the final payments are the same and equations (5.1) can be solved by not changing the portfolio at these instants. Therefore, we can always find a specific option that enables us to replicate a specific payoff scheme.

In Table 2 we give the initial costs of the dynamic self-financing portfolio strategies using a call option with an exercise price of 11 to replicate the stop-loss strategy and the alternative efficient strategy. Also, the initial costs of the lock-in strategy and its efficient alternative are given when a put option with an exercise price of 23 is used to replicate the final payoffs. Table 2 demonstrates that the efficient strategies once again are no longer efficient even if transaction costs are only $0.5 \%$. More importantly, if we compare Tables 1 and 2 we see that for all strategies the use of options dramatically decreases the initial costs if the Iransaction costs on stocks and options are the same, i.e. if $k=l$. Often, the proportional transaction costs on stocks are lower than the proportional transaction costs on options and one would be inclined to use slocks instead of options to replicate a payoff scheme. If we compare row $l=2 \%$ from Table 2 with row $k=1 \%$ from Table 1, we see that the row in Table 1 has higher initial costs for all four strategies. Thus, even if transaction costs on single trades in options are twice as high as the transaction costs on single trades in stocks, it is still cheaper to use options for the replicating strategies. Hence, options really allow investors to reduce portfolio management costs.

We have only used dynamic self-financing portfolio strategies that use just one option to lower the initial costs and at the same time maintain the payoff distribution. In the real world however, one can use strategies involving not just one stock or one option, but one can replicate with stocks and several put and call options which differ in exercise price and maturity dates. Hence, the optimal strategy will not only have lower costs than the strategies based on stocks, but also than the strategies based on single options. Therefore, if there are transaction costs, options will enable investors to implement dynamic self-financing portfolio strategies, that reduce initial costs while maintaining the payoff distribution. For large transaction costs this would not be possible if only stocks were available to set up the strategy.

\section{Conclusion}

In this paper we investigated the effect of transaction costs on dynamic portfolio strategies. We showed that even with a modest level of transaction costs of $0.5 \%$, simple trading strategies such as stop-loss and lockin have a better return distribution than more complex strategies that would be efficient if there were no transaction costs. Furthermore, we showed that the use of options considerably reduces the initial costs of both simple and efficient strategies. This conclusion holds even if the transaction costs on options are twice as high as the transaction costs on stocks. Due to the fact that all calculations require the use of an expanded binomial tree we could only use the binomial model with 18 trading intervals. In further research one should focus on the same results for binomial models with more trading intervals or on similar results for the continuous time model, as was done by Leland (1985), for the replication of options. This will enable us to understand the significance of options for complicated dynamic portfolio strategies better. Especially, a clear view on the cost efficiency of the use of options in an environment with transaction costs is important.

\section{Appendix A}

The text of this appendix illustrates that if a dynamic self-financing strategy $\left(\Delta^{\prime}, B^{\prime}\right)$ stochastically dominates another self-financing strategy $(\Delta, B)$ in the no-transaction costs case, then there exists a third dynamic self-financing strategy $\left(\Delta^{\prime \prime}, B^{\prime \prime}\right)$ with nonnegative payoffs in all states and a positive payoff in at least one state, such that $G(\Delta, B)=G\left(\Delta^{\prime}-\Delta^{\prime \prime}, B^{\prime}-\right.$ $\left.B^{\prime \prime}\right)$.

Let $x_{0}=\inf \left\{y \mid G(\Delta, B)(y)>G\left(\Delta^{\prime}, B^{\prime}\right)(y)\right\}$ and define Dif $=2^{n}\left(G(\Delta, B)\left(x_{0}\right)-G\left(\Delta^{\prime}, B^{\prime}\right)\left(x_{0}\right)\right)$, with $n$ being the number of trading intervals in the binomial tree. Because we assumed that all states have the same probability, each of the $2^{n}$ final states has probability $2^{-n}$. Therefore, Dif is the number of states that have payoff $x_{0}$. Let $x>x_{0}$ be the next place where there is a jump in the step-function $G\left(\Delta^{\prime}, B^{\prime}\right)$ and let $\omega$ be one of the states that causes this jump. Hence $\Delta^{\prime}(\omega) S(\omega)+B^{\prime}(\omega)=x$. Now reduce the payoff function of $\left(\Delta^{\prime}, B^{\prime}\right)$ in just this one state with $x-x_{0}$ to $x_{0}$. Let the unique new 
strategy be denoted by $(\tilde{A}, \tilde{B})$. If Dif $>1$, then $x_{0}=\inf \left\{y \mid G(\Delta, B)(y)>G\left(\Delta^{\prime}, B^{\prime}\right)(y)\right\}$, but $2^{n}\left(G(\Delta, B)\left(x_{0}\right)-G(\tilde{\Delta}, \tilde{B})\left(x_{0}\right)\right)=\operatorname{Dif}-1$ and we can again apply the above procedure and update $(\tilde{\Delta}, \tilde{B})$ once more until $2^{n}\left(G(\Delta, B)\left(x_{0}\right)-\right.$ $\left.G(\tilde{\Delta}, \tilde{B})\left(x_{0}\right)\right)=0$. Then we find the next $x_{0}^{\prime}=\inf \{y \mid$ $G(\Delta, B)(y)>G(\tilde{\Delta}, \tilde{B})(y)\}$. It is easy to check that $x_{0}^{\prime}>x_{0}$ and the $x_{0}$ 's can only be final payoffs of $G(\Delta, B)$ of which there are finitely many. Hence after finitely many steps, we have reduced the payoff of $\left(\Delta^{\prime}, B^{\prime}\right)$ in several states such that $G(\Delta, B)=$ $G(\tilde{A}, \tilde{B})$. Now let $\left(\Delta^{\prime \prime}, B^{\prime \prime}\right)=\left(\Delta^{\prime}-\tilde{A}, B^{\prime}-\tilde{B}\right)$ which has all positive payoffs.

\section{Appendix B}

In this appendix we show that the problem of finding the dynamic self-financing trading strategy $\left(\Delta^{\prime}, B^{\prime}\right)$ in a world with transaction costs that has the lowest initial costs among the stategies that dominate $G(\Delta, B)$ can be formulated as a mixed-integer programming problem. Let $g_{i}(i=1, \ldots, m)$ be the different possible payoffs of $G(\Delta, B)$ in the final states and let $r_{i}$ be the number of final states with payoff $g_{i}$. Let $t=0, \ldots, T$ denote the steps in the tree, then we have $j=1, \ldots, 2^{t}$ states of the world in step $t$. In the final step $t=T$, we have $2^{T}$ final states of the world, hence $\sum_{i=1}^{m} r_{i}=$ $2^{T}$. If we number the states in step $t$ from top to bottom (as we did in Fig. 1 for the final states), then we can reach from state $(t, j)$ the state $(t+1,2 j-1)$ if we go one step up, and state $(t+1,2 j)$ if we go one step down. Finally, the stock price is given by $S_{t j}$ $\left(t=0, \ldots, T ; j=1, \ldots, 2^{t}\right)$ in every state.

Decision variables are the real variables $\Delta_{t j}^{\prime}, B_{t j}^{\prime}(t=$ $\left.0, \ldots, T ; j=1, \ldots, 2^{t}\right)$ and the zero-one variables $y_{i j}\left(i=1, \ldots, m ; j=1, \ldots, 2^{T}\right)$. The optimisation problem can be formulated as follows

$\min \Delta_{01}^{\prime} S_{01}+B_{01}^{\prime}$

subject to

$$
\begin{gathered}
\left(\Delta_{t j}^{\prime}-\Delta_{t+1,2 j-1}^{\prime}\right) S_{t+1,2 j-1}+r B_{t j}^{\prime}-B_{t+1,2 j-1}^{\prime} \\
\geqslant k\left|\Delta_{t, j}^{\prime}-\Delta_{t+1,2 j-1}^{\prime}\right| S_{t+1,2 j-1} \\
\quad\left(t=0, \ldots, T-2 ; j=1, \ldots, 2^{t}\right)
\end{gathered}
$$

$$
\begin{aligned}
& \left(\Delta_{t j}^{\prime}-\Delta_{t+1,2 j}^{\prime}\right) S_{t+1,2 j}+r B_{t j}^{\prime}-B_{t+1,2 j}^{\prime} \\
& \geqslant k\left|\Delta_{t j}^{\prime}-\Delta_{t+1,2 j}^{\prime}\right| S_{t+1,2 j} \\
& \left(t=0, \ldots, T-2 ; j=1, \ldots, 2^{\prime}\right), \\
& \sum_{i=1}^{m} y_{i j}=1 \quad\left(j=1, \ldots, 2^{T}\right), \\
& \sum_{j=1}^{2^{T}} y_{i j} \geqslant r_{i} \quad(i=1, \ldots, m), \\
& \Delta_{T-1, j}^{\prime} S_{T, 2 j-1}+r B_{T-1, j}^{\prime} \geqslant \sum_{i=1}^{m} g_{i} y_{i, 2 j-1} \\
& \left(j=1, \ldots, 2^{T-1}\right), \\
& \Delta_{T-1, j}^{\prime} S_{T, 2 j}+r B_{T-1, j}^{\prime} \geqslant \sum_{i=1}^{m} g_{i} y_{i, 2 j} \\
& \left(j=1, \ldots, 2^{T-1}\right), \\
& y_{i, j} \in\{0,1\} \quad\left(i=1, \ldots, m ; j=1, \ldots, 2^{T}\right) .
\end{aligned}
$$

Conditions (B.1) and (B.2) are the self-financing conditions in state $(t, j)$ for an up- and a down-move respectively (compare with (3.3)). (B.3) assigns each of the final states $j$ to one of the payoffs $g_{i}$. (B.4) guarantees that there are at least $r_{i}$ states with this payoff. Finally, (B.5) and (B.6) require that the final portfolio's $\Delta_{T-1, j}^{\prime}, B_{T-1, j}^{\prime}$ ) will match the required payoff in the last period $T$ after an up- and a down-move respectively.

\section{Acknowledgments}

The authors are grateful to Frank Debets for research assistance and to Angelien Kemna for helpful suggestions. This research was sponsored by the Economics Research Foundation, which is part of the Netherlands Organisation for Scientific Research (NWO).

\section{References}

Bensaid, B., Lesne, J.P., Pagès, H., and Scheinkman, J. (1992), "Derivative asset pricing with transaction costs", Mathematical Finance $2 / 2,63-86$. 
Boyle, P.P., and Vorst. T. (1992), "Option pricing in discrete time with transaction costs", Journal of Finance, 47/1, 271-293.

Cox, J.C., Ross, S.A., and Rubinstein, M. (1979), "Option pricing: A simplified approach", Journal of Financial Economics 7, 229-263.

Dothan, M.U. (1990), Prices in Financial Markets, Oxford University Press, New York.

Dybvig, P.H. (1988a), "Distributional analysis of portfolio choice", Journal of Business 61/3, 369-393.

Dybvig, P.H. (1988b), "Inefficient dynamic portfolio strategies or how to throw away a million dollars in the stock market", The
Review of Financial Studies 1/1, 67-88.

Edirisinghe, C., Naik, V., and Uppal, R. (1993), "Optimal replication of options with transaction costs and trading restrictions", Journal of Financial and Quantitative Analysis $28 / 1,117-138$

Ingersoll, Jr., J.E. (1987), Theory of Financial Decision Making, Rowman \& Littlefield, Totowa, NJ.

Leland, H.E. (1985), "Option pricing and replication with transactions costs", Journal of Finance 40/5, 1283-1301.

Roll, R. (1992), "A mean/variance analysis of tracking error", Journal of Portfolio Management, Summer, 13-22. 